\title{
Coherent Radiation by Quantum Dots and Magnetic Nanoclusters
}

\author{
V.I. Yukalov* and E.P. Yukalova ${ }^{\dagger}$ \\ *Bogolubov Laboratory of Theoretical Physics, Joint Institute for Nuclear Research, Dubna 141980, Russia \\ ${ }^{\dagger}$ Laboratory of Information Technologies, Joint Institute for Nuclear Research, Dubna 141980, Russia
}

\begin{abstract}
The assemblies of either quantum dots or magnetic nanoclusters are studied. It is shown that such assemblies can produce coherent radiation. A method is developed for solving the systems of nonlinear equations describing the dynamics of such assemblies. The method is shown to be general and applicable to systems of different physical nature. Despite mathematical similarities of dynamical equations, the physics of the processes for quantum dots and magnetic nanoclusters is rather different. In a quantum dot assembly, coherence develops due to the Dicke effect of dot interactions through the common radiation field. For a system of magnetic clusters, coherence in the spin motion appears due to the Purcell effect caused by the feedback action of a resonator. Self-organized coherent spin radiation cannot arise without a resonator. This principal difference is connected with the different physical nature of dipole forces between the objects. Effective dipole interactions between the radiating quantum dots, appearing due to photon exchange, collectivize the dot radiation. While the dipolar spin interactions exist from the beginning, yet before radiation, and on the contrary, they dephase spin motion, thus destroying the coherence of moving spins. In addition, quantum dot radiation exhibits turbulent photon filamentation that is absent for radiating spins.
\end{abstract}

Keywords: coherent radiation, quantum dots, magnetic nanoclusters, spin reversal PACS: $75.40 . \mathrm{Gb}, 75.40 . \mathrm{Mg}, 75.50 . \mathrm{Xx}, 75.60 . \mathrm{Jk}, 75.75 . \mathrm{Jn}, 78.67 . \mathrm{Bf}, 78.67 . \mathrm{Hc}$

\section{INTRODUCTION}

The aim of this article is to demonstrate the feasibility of employing nano-objects, such as quantum dots and magnetic nanoclusters, as sources of coherent radiation. The assemblies of such nano-objects have many features common with the systems of resonance atoms that are in the basis of laser physics [1,2], hence, these nanoobjects also could be used for creating collective sources of coherent radiation, similar to resonance atoms.

Though both, quantum dots and magnetic nanoclusters, remind finite-level atoms and show several properties common for atoms, exhibiting similarities in the dynamics of dots and clusters, there are as well important differences caused by the different physical nature of the nano-objects. We concentrate our attention on the collective effects in the dynamics of quantum dots and magnetic nanoclusters. We emphasize the necessity of employing microscopic description of the processes, where all system parameters are well defined. The use of phenomenological equations often leads to wrong conclusions that are widespread in literature. The theory, based on microscopic Hamiltonians, allows us to clearly understand the physical origins of and the necessary conditions for achieving coherent dynamics. The microscopic quantum picture is especially important for describing the self-organized birth of coherence from initial chaotic fluctuations. Such a self-organized coherence cannot be described by phenomenological equations of semi-classical type.

Despite the very different physical nature of quantum dots and magnetic nanoclusters, their dynamics can be reduced to mathematically similar equations that can be analyzed by the general method of scale separation. At the same time, because of their different physical nature, the physics of their collective dynamics is very different. We emphasize that the origins of collective phenomena in quantum dots and magnetic nanoclusters are principally different. In a quantum-dot system, coherence develops due to the photon exchange through the common radiation field, that is, due to the Dicke effect [3]. While in an assembly of magnetic nanoclusters, the Dicke effect is impossible and coherence can arise only through a resonator feedback field, that is, due to the Purcell effect [4].

\section{QUANTUM DOTS}

Electrons in quantum dots are confined in all three spatial dimensions, which makes their spectrum discrete [5-8]. Exciting an electron from the ground-state level creates a hole. The interacting pair of an electron and a hole forms an exciton, whose recombination is accompanied by electromagnetic radiation, in a close analogy with atomic radiation.

There exists a variety of quantum dots, for instance, the dots based on self-assembled heterostructures, 
such as InAs/GaAs, InGaAs/GaAs, InGaAs/AlGaAs, GaInAsP/InP, InAs/InP, InAs/GaInAs, AlInAs/AlGaAs, InP/GaInP, AlGaAs/GaAs, CdSe/ZnSe, and so on. In each dot there can be between 2 to $10^{5}$ electrons.

The characteristic lengths related to quantum dots are as follows. The dot size is $r_{d o t} \sim 10^{-7}-10^{-6} \mathrm{~cm}$, the interdot distance, $a \sim 10^{-5}-10^{-4} \mathrm{~cm}$, radiation wavelength, $\lambda \sim 10^{-4} \mathrm{~cm}$, the collection of quantum dots, forming a kind of a laser, has the radius and length $R, L \sim 10^{-3}-10^{-2} \mathrm{~cm}$, which makes the volume $V=$ $\pi R^{2} L \sim 10^{-8}-10^{-5} \mathrm{~cm}^{3}$. The dot density in a laser is $\rho \sim 10^{13}-10^{17} \mathrm{~cm}^{-3}$. Hence the total number of dots is $N=\rho V \sim 10^{5}-10^{12}$.

The longitudinal relaxation time is due to electronphonon coupling that is suppressed at low temperatures, so that, at helium temperatures, $T_{1} \sim 10^{-9} \mathrm{~s}$. The homogeneous dephasing time is $T_{2} \sim 10^{-13}-10^{-12} \mathrm{~s}$. For high-quality self-assembled heterostructures, the inhomogeneous broadening is of the same order as the homogeneous broadening, so that $T_{2}^{*} \sim 10^{-13}-10^{-12} \mathrm{~s}$.

The transition frequency is $\omega_{0} \sim 10^{15} \mathrm{~Hz}$. The natural width is $\gamma_{0} \sim 10^{10} \mathrm{~Hz}$, the longitudinal relaxation width, $\gamma_{1} \sim 10^{9} \mathrm{~Hz}$, the homogeneous broadening, $\gamma_{2} \sim 10^{12}-$ $10^{13} \mathrm{~Hz}$, and the inhomogeneous broadening is $\gamma_{2}^{*} \sim$ $10^{12}-10^{13} \mathrm{~Hz}$. To select and enhance the chosen mode, the sample is placed into a high-quality resonator cavity.

Since the wavelength is much larger than the dot size, $\lambda \gg r_{d o t}$, the interaction of a dot with electromagnetic field can be treated in the dipole approximation. The wavelength is also larger than the interdot distance, $\lambda \gg$ $a$, which tells us that there should exist essential interaction between dots. And the wavelength is much shorter than the sample sizes, $\lambda \ll R, L$, because of which the point-sample approximation is not allowed.

The possibility of coherent quantum dot radiation has been mentioned in Ref. [9] and discussed in detail in Ref. [10]. The microscopic Hamiltonian for an ensemble of radiating quantum dots in a semiconductor matrix inside a resonator cavity [10] is the sum

$$
\hat{H}=\hat{H}_{d}+\hat{H}_{f}+\hat{H}_{d f}+\hat{H}_{m f} .
$$

Here the dot Hamiltonian is

$$
\hat{H}_{d}=\sum_{i=1}^{N} \omega_{0}\left(\frac{1}{2}+S_{i}^{z}\right),
$$

with $\omega_{0}$ being the carrying transition frequency and $S_{i}^{z}$, a pseudospin operator of population imbalance. The field Hamiltonian is

$$
\hat{H}_{f}=\frac{1}{8 \pi} \int\left(\mathbf{E}^{2}+\mathbf{H}^{2}\right) d \mathbf{r},
$$

where $\mathbf{E}$ is electric field, $H=\nabla \times \mathbf{A}$ is magnetic field, $\mathbf{A}$ is vector potential satisfying the Coulomb calibration $\nabla \cdot \mathbf{A}=0$. The dot-field interaction is given by the Hamiltonian

$$
\hat{H}_{d f}=-\sum_{i=1}^{N}\left(\frac{1}{c} \mathbf{J}_{i} \cdot \mathbf{A}_{i}+\mathbf{P}_{i} \cdot \mathbf{E}_{i 0}\right)
$$

with the transition current

$$
\mathbf{J}_{i}=i \omega_{0}\left(\mathbf{d} S_{i}^{+}-\mathbf{d}^{*} S_{i}^{-}\right)
$$

and transition polarization

$$
\mathbf{P}_{i}=\mathbf{d} S_{i}^{+}+\mathbf{d}^{*} S_{i}^{-}
$$

where $\mathbf{A}_{i}=\mathbf{A}\left(\mathbf{r}_{i}, t\right), \mathbf{E}_{i 0}$ is the cavity seed field, $\mathbf{d}$, transition dipole, and $S_{i}^{ \pm}$is a pseudospin ladder operator. The cavity is filled by a semiconducting material interacting with the radiation field through the Hamiltonian

$$
\hat{H}_{m f}=-\frac{1}{c} \int \mathbf{j}_{m a t} \cdot \mathbf{A} d \mathbf{r},
$$

where $\mathbf{j}_{\text {mat }}$ is a fluctuating local-density current of the semiconductor matrix.

We eliminate the field variables by writing down the Heisenberg equations of motion, solving the d'Alembert equation for $\mathbf{A}_{i}$, expressing it through the pseudospin operators, and substituting the d'Alembert solution to the pseudospin equations of motion [10, 11]. Then we average the latter equations defining the statistical average

$$
\left\langle S_{i}^{\alpha}(t)\right\rangle \equiv \operatorname{Tr} \hat{\rho}(0) S_{i}^{\alpha}(t) .
$$

The resulting equations are written for the transition function

$$
u_{i} \equiv 2\left\langle S_{i}^{-}\right\rangle
$$

coherence intensity

$$
w_{i} \equiv \frac{2}{N} \sum_{j(\neq i)}\left\langle S_{i}^{+} S_{j}^{-}+S_{j}^{+} S_{i}^{-}\right\rangle,
$$

and the population imbalance

$$
s_{i} \equiv 2\left\langle S_{i}^{z}\right\rangle
$$

\section{SCALE SEPARATION}

The evolution equations for the above variables acquire the form

$$
\frac{d u_{i}}{d t}=f_{\text {iu }}, \quad \frac{d w_{i}}{d t}=f_{i w}, \quad \frac{d s_{i}}{d t}=f_{i s},
$$

with the right-hand sides depending on the variables $u_{i}, w_{i}, s_{i}, \xi_{i}$, and time $t$. Here $\xi_{i}$ is a random field caused by vacuum fluctuations, dipole fluctuations, and 
semiconductor-matrix current fluctuations. We notice that when the widths $\gamma_{0}, \gamma_{1}, \gamma_{2}$, and $\gamma_{2}^{*}$, all of which are smaller than $\omega_{0}$, tend to zero, then $f_{i w}$ and $f_{i s}$ also tend to zero, while $f_{i u}$ remains finite. This allows us to classify the variable $u_{i}$ as fast and the variables $w_{i}, s_{i}$ as slow. Therefore, for solving these evolution equations, we can resort to the scale separation approach [11-15]. The solution proceeds as follows. We solve the equation for the fast variable $u_{i}$, keeping the slow variables fixed. This solution is substituted into the right-hand sides of the equations for the slow variables. Then these right-hand sides are averaged over time and over the random fluctuations $\xi_{i}$, again keeping the slow variables fixed, which corresponds to the rule

$$
\bar{f} \equiv \lim _{\tau \rightarrow \infty} \frac{1}{\tau} \int_{0}^{\tau}\langle\langle f(t)\rangle\rangle d t
$$

where the double angle brackets denote the averaging over the stochastic fluctuations. Thus we come to the equations

$$
\frac{d w_{i}}{d t}=\bar{f}_{i w}, \quad \frac{d s_{i}}{d t}=\bar{f}_{i s}
$$

defining the guiding centers of the slow variables.

\section{DOT RADIATION}

The total radiation intensity of quantum dots, averaged over fast fluctuations, can be represented [16] as the sum of two terms,

$$
I(t)=I_{\text {inc }}(t)+I_{\text {coh }}(t),
$$

where the first term is the incoherent radiation intensity

$$
I_{\text {inc }}(t)=\frac{1}{2} \omega_{0} \gamma_{0}(1+s) N
$$

while the second term is the coherent radiation intensity

$$
I_{c o h}(t)=\omega_{0} \gamma_{0} \varphi_{s} w N^{2}
$$

with $\varphi_{s}$ being a shape factor [1] and $s$ and $w$ being the spatial averages over the coherence volume [10].

The spatial distribution of radiation depends on the value of the Fresnel number $F \equiv R^{2} / \lambda L$. For $F<1$, the radiating beam is uniform. In the range $1<F<10$, the radiation is separated into a few Gauss-Laguerre modes dictated by the sample geometry. And when $F>10$, the effect of turbulent photon filamentation [17-21] appears. Then the radiation beam separates into the large number

$$
N_{f}=3.3 F
$$

of filaments with radius

$$
r_{f}=0.3 \sqrt{\lambda L}
$$

According to Eq. (13), the coherent radiation is formally proportional to the number of radiators squared, $N^{2}$. However, since $\lambda \ll R, L$, it is necessary to take into account the shape factor that, depending on either the pencil or disk geometry of the sample, behaves as

$$
\varphi_{s} \propto \begin{cases}N^{-1 / 3} & (\text { pencil }) \\ N^{-2 / 3} & (\text { disk })\end{cases}
$$

Respectively, the coherent radiation exhibits the dependence on the dot number as

$$
I_{c o h} \propto\left\{\begin{array}{ll}
N^{5 / 3} & (\text { pencil }) \\
N^{4 / 3} & (\text { disk })
\end{array} .\right.
$$

The temporal evolution of radiation consists of the following stages. The very first and short is the interaction stage, when the dots start radiating, but have had yet no time for initiating mutual interactions:

$$
0<t<t_{\text {int }} \quad \text { (interaction stage) } .
$$

Here the interaction time is

$$
t_{\text {int }}=\frac{a}{c} \sim 10^{-15}-10^{-14} \mathrm{~s}
$$

The second is the quantum stage, when the dots have started interacting with each other through the common radiation field, but radiate yet chaotically, without noticeable mutual correlations:

$$
t_{\text {int }}<t<t_{\text {coh }} \quad \text { (quantum stage) } .
$$

The stage lasts till the coherence time

$$
t_{c o h}=\frac{T_{2}}{2 g s_{0}} \sim 10^{-14}-10^{-13} \mathrm{~s},
$$

in which $s_{0} \equiv s(0)$ and the coupling parameter

$$
g=\frac{\rho \gamma_{0} \lambda^{2} L}{4 \pi \gamma_{2}}
$$

characterizes the effective dot interactions through the photon exchange. With the considered parameters, the value of $g$ can be as high as $g \sim 10^{3}$ for the dot density $\rho \sim 10^{17} \mathrm{~cm}^{-3}$. The interaction and quantum stages cannot be described by the semiclassical approximation, but require microscopic quantum description.

After the coherence time, the dots become correlated and radiate coherently in the temporal interval

$$
t_{\text {coh }}<t<T_{2} \quad \text { (coherent stage) }
$$


till they are dephased at the dephasing time $T_{2} \sim 10^{-13}-$ $10^{-12} \mathrm{~s}$. In the case of pure superradiance, when the coherent radiation is self-organized, the radiation peak occurs at the delay time $t_{0} \sim 5 t_{\text {coh }}$ and the superradiance pulse duration is $t_{p} \sim 2 t_{c o h}$.

The next is the relaxation stage, when the radiation can yet be noticeable, but already not coherent and quickly diminishing:

$$
T_{2}<t<T_{1} \quad \text { (relaxation stage) } .
$$

The radiation practically vanishes at the longitudinal relaxation time $T_{1} \sim 10^{-9} \mathrm{~s}$.

Finally, after $T_{1}$, there is almost no radiation, but just some weak splashes caused by random fluctuations. This can be called the stationary stage:

$$
t>T_{1} \quad(\text { stationary stage) } .
$$

Figure 1 presents the typical behavior of the coherence intensity and population imbalance as functions of time. These functions are smoothed by averaging over fast oscillations of the period $2 \pi / \omega_{0}$.

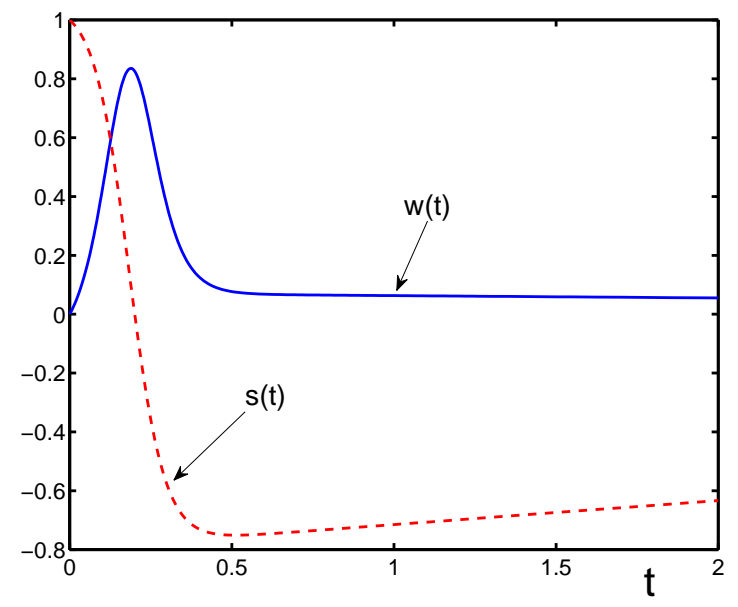

FIGURE 1. Pure dot superradiance, with initial conditions $w_{0}=0, s_{0}=1$. Coherence intensity (solid line) and population difference (dashed line) as functions of time (in units of $T_{2}$ ) for $\gamma_{1}=0.003 \gamma_{2}$ and $g=10$.

If the dots are subject to stationary nonresonant pumping, characterized by the pumping parameter $\gamma_{1}^{*}$, then the regime of pulsing superradiance develops, exhibiting several superradiant pulses, as is shown in Fig. 2.

Figures 1 and 2 correspond to pure dot superradiance, when there are no external pulses imposing initial coherence on the sample, so that $w_{0} \equiv w(0)=0$. The radiation can also be pushed by a coherent initial pulse yielding $w_{0}>0$. Then one has the triggered dot superradiance, with essentially shortened delay time $t_{0}$.

To estimate the radiation intensity in dimensional units (in Watts), we take the typical dot parameters mentioned

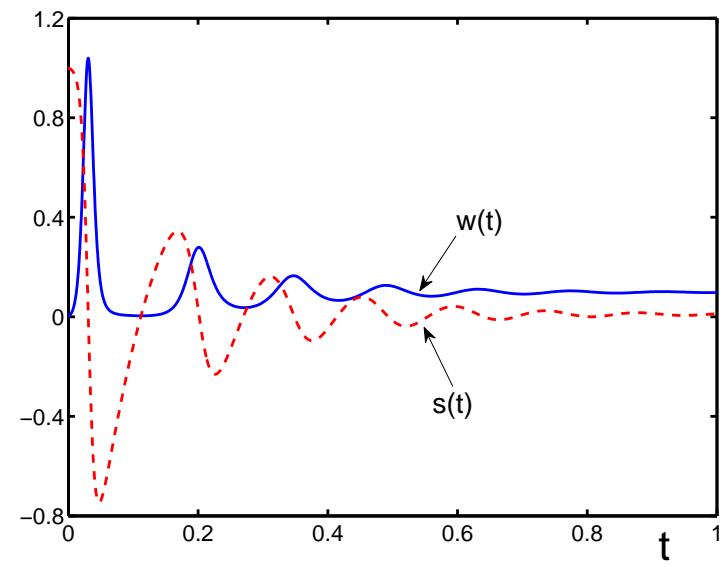

FIGURE 2. Pulsing dot superradiance, under initial conditions $w_{0}=0, s_{0}=1$, with the pumping parameter $\gamma_{1}^{*}=10 \gamma_{2}$ and coupling parameter $g=100$. Coherent intensity (solid line) and population difference (dashed line).

above, which gives the incoherent radiation intensity $I_{\text {inc }} \sim 10^{-9} \mathrm{~N} \mathrm{~W}$ and the coherent radiation intensity $I_{c o h} \sim 10^{-9} \varphi_{s} N^{2} \mathrm{~W}$. The shape factor for the pencil and disk geometry is

$$
\varphi_{s} \simeq \begin{cases}\frac{3 \lambda}{8 L}, & \frac{R}{L} \ll 1 \\ \frac{3}{8}\left(\frac{\lambda}{\pi R}\right)^{2}, & \frac{L}{R} \ll 1,\end{cases}
$$

which, with the given parameters, translates into

$$
\varphi_{s} \simeq \begin{cases}10^{-2}, & \frac{R}{L} \ll 1 \\ 10^{-3}, & \frac{L}{R} \ll 1 .\end{cases}
$$

The number of dots that can radiate coherently is

$$
N_{c o h}=\rho \pi \lambda^{2} L .
$$

For the dot density $\rho \sim 10^{17} \mathrm{~cm}^{-3}$, we have $N \sim 10^{7}$. Then, for a pencil-like sample of $N \sim 10^{7}$ dots, we get the incoherent intensity $I_{\text {inc }} \sim 10^{-2} \mathrm{~W}$ and the coherent intensity $I_{c o h} \sim 10^{3} \mathrm{~W}$. The superradiant pulse duration is $10^{-13} \mathrm{~s}$.

\section{MAGNETIC NANOCLUSTERS}

Under magnetic nanoclusters, we here keep in mind three possible types of such objects, polarized nanomolecules, magnetic nanomolecules, and magnetic nanoclusters as such. Polarized nanomolecules can be put together, forming sufficiently large solids. Magnetic nanomolecules can form crystals with well defined crystalline lattice. 
Magnetic nanoclusters are the clusters that can possess a total nonzero spin, thus enjoying nonzero magnetization. The sizes of such clusters are of nanoscale, having typical radii not larger than the coherence radius, since only then a cluster presents a single magnetic domain with a nonzero total spin. The larger clusters separate into several domains, so that the total spin becomes zero. There exists a variety of different magnetic nanoclusters [22-26]. It is possible to distinguish three main classes of such magnetic objects.

(i) Polarized nanomolecules. These are large molecules that do not possess nonzero spin in their ground state. However, they contain many hydrogen atoms whose protons can be polarized and, being kept a low temperature $T<1 \mathrm{~K}$, the polarization remains frozen for very long time. There exist many such molecules, among which we can mention propanediol $\mathrm{C}_{3} \mathrm{H}_{8} \mathrm{O}_{2}$, butanol $\mathrm{C}_{4} \mathrm{H}_{9} \mathrm{OH}$, and ammonia $\mathrm{NH}_{3}$. Materials, formed by such molecules, are characterized by the density of protons, each having spin $S=1 / 2$.

(ii) Magnetic nanomolecules. Such molecules possess nonzero total spin, due to electrons, in their ground state. At low temperature, below the blocking temperature $T_{B} \sim 1-10 \mathrm{~K}$, the spin can be fixed for long time. There is a number of such magnetic molecules. The most often considered are the molecules denoted as $\mathrm{Mn}_{12}$ and $\mathrm{Fe}_{8}$, whose complete formulas are

$$
\begin{gathered}
\mathrm{Mn}_{12} \mathrm{O}_{12}\left(\mathrm{CH}_{3} \mathrm{COO}\right)_{16}\left(\mathrm{H}_{2} \mathrm{O}\right)_{4}\left(2 \mathrm{CH}_{3} \mathrm{COOH}\right) 4 \mathrm{H}_{2} \mathrm{O}, \\
{\left[\mathrm{Fe}_{8} \mathrm{O}_{2}(\mathrm{OH})_{12} \operatorname{tacn}_{6}\right]^{+8},}
\end{gathered}
$$

where tacn stands for triazacyclononane. The groundstate spin of these molecules is $S=10$. Magnetic nanomolecules can form crystals with a good crystalline lattice.

(iii) Magnetic nanoclusters. Truly magnetic nanoclusters are composed of magnetic atoms or molecules correlated by exchange interactions. For instance, such clusters can be formed by the atoms of $\mathrm{Fe}, \mathrm{Ni}$, or $\mathrm{Co}$, or by oxides, such as $\mathrm{NiO}, \mathrm{Fe}_{2} \mathrm{O}_{3}$, and $\mathrm{NiFe}_{2} \mathrm{O}_{4}$. Below the blocking temperature $T_{B} \sim 10-100 \mathrm{~K}$, the total spin of each nanocluster, reaching $S \sim 10^{2}-10^{5}$, can be kept frozen for very long time.

Magnetic nanoclusters find numerous applications in magnetic chemistry, biomedical imaging, cancer treatment, genetic engineering, waste cleaning, information storage, and quantum computing [22-26].

The generic Hamiltonian for a system of magnetic nanoclusters writes as

$$
\hat{H}=\sum_{i} \hat{H}_{i}+\frac{1}{2} \sum_{i \neq j} \hat{H}_{i j},
$$

where the index $i=1,2, \ldots, N$ enumerates nanoclusters. The single-cluster Hamiltonian is

$$
\hat{H}_{i}=-\mu_{i} \mathbf{B} \cdot \mathbf{S}_{i}-D\left(S_{i}^{z}\right)^{2}+D_{2}\left(S_{i}^{x}\right)^{2}+
$$

$$
+D_{4}\left[\left(S_{i}^{x}\right)^{2}\left(S_{i}^{y}\right)^{2}+\left(S_{i}^{y}\right)^{2}\left(S_{i}^{z}\right)^{2}+\left(S_{i}^{z}\right)^{2}\left(S_{i}^{x}\right)^{2}\right],
$$

where the first term is the Zeeman energy and the following terms describe magnetic anisotropy. The nanocluster interactions are given by the Hamiltonian

$$
\hat{H}_{i j}=\sum_{\alpha \beta} D_{i j}^{\alpha \beta} S_{i}^{\alpha} S_{j}^{\beta},
$$

with the dipolar tensor

$$
D_{i j}=\frac{\mu_{i} \mu_{j}}{r_{i j}^{3}}\left(\delta_{\alpha \beta}-3 n_{i j}^{\alpha} n_{i j}^{\beta}\right),
$$

in which $r_{i j} \equiv\left|\mathbf{r}_{i j}\right|, \mathbf{n}_{i j} \equiv \mathbf{r}_{i j} / r_{i j}$, and $\mathbf{r}_{i j} \equiv \mathbf{r}_{i}-\mathbf{r}_{j}$.

The total magnetic field

$$
\mathbf{B}=B_{0} \mathbf{e}_{z}+H \mathbf{e}_{x}
$$

consists of an external magnetic field $B_{0}$ and of the feedback field of a resonant electric circuit $[25,26]$. The resonator feedback field is described by the Kirchhoff equation

$$
\frac{d H}{d t}+2 \gamma H+\omega^{2} \int_{0}^{t} H\left(t^{\prime}\right) d t^{\prime}=-4 \pi \eta \frac{d m_{x}}{d t},
$$

where $\gamma$ is resonator damping, $\omega$, resonator natural frequency, $\eta$ is a filling factor and

$$
m_{x} \equiv \frac{1}{V} \sum_{j} \mu_{j}\left\langle S_{j}^{x}\right\rangle
$$

Similarly to Eqs. (8) to (10), we introduce the dynamical variables, the transverse component

$$
u \equiv \frac{1}{S N} \sum_{j=1}^{N}\left\langle S_{j}^{-}\right\rangle
$$

the coherence intensity

$$
w \equiv \frac{1}{S^{2} N^{2}} \sum_{i \neq j}^{N}\left\langle S_{i}^{+} S_{j}^{-}\right\rangle,
$$

and the spin polarization

$$
s \equiv \frac{1}{S N} \sum_{j=1}^{N}\left\langle S_{j}^{z}\right\rangle .
$$

The evolution equations for these variables are obtained from the related Heisenberg equations for spins, with the feedback field given by the Kirhhoff equation (26). The following steps of solving these equations are analogous to those corresponding to the solution of pseudospin equations for quantum dots. These equations have been analyzed for polarized molecules [11-15, 25, 27], magnetic nanomolecules [25, 28-32], and for magnetic 
nanoclusters [26, 33, 34]. The results obtained by the scale separation approach [11-15, 25] have been compared with direct numerical simulations [35-37] of the evolution equations for spins, both ways being in good agreement with each other.

Here we illustrate the solution to the spin equations of motion for the parameters typical of such nanoclusters as those composed of $\mathrm{Fe}, \mathrm{Ni}$, and $\mathrm{Co}$. These nanoclusters are characterized by the following parameters. The Zeeman frequency is taken as $\omega_{0} \sim 10^{10}-10^{12} \mathrm{~Hz}$, which translates into the wavelength $\lambda \sim 0.1-10 \mathrm{~cm}$. The sample of $N \sim 10^{14}-10^{20}$ nanoclusters has the linear sizes comparable with the wavelength, because of which there is no the filamentation effect that occurs for quantum dots. The nanocluster density in the sample is $\rho \sim 10^{20} \mathrm{~cm}^{-3}$. The intercluster distance is $a \sim 10^{-7} \mathrm{~cm}$. The feedback rate, due to the coupling of the nanoclusters with a resonator, is $\gamma_{0} \sim 10^{10} \mathrm{~s}^{-1}$. Typical anisotropy parameters are given by the relations

$$
\frac{D}{\hbar \gamma_{0}} \sim 10^{-3}, \quad \frac{D_{2}}{\hbar \gamma_{0}} \sim 10^{-3}, \quad \frac{D_{4}}{\hbar \gamma_{0}} \sim 10^{-10} .
$$

Below the blocking temperature $T_{B} \sim 10 \mathrm{~K}$, the total spin $S \sim 10^{3}$ is frozen, so that the longitudinal relaxation time $T_{1}$ reaches years. The transverse dephasing time $T_{2} \sim 10^{-10} \mathrm{~s}$ is caused by dipole spin interactions. The coupling parameter, defining the effective spin-resonator interaction, is $g \sim \omega_{0} / \gamma \sim 1-100$.

Figure 3 demonstrates the temporal behaviour of the coherence intensity and spin polarization in the regime of pure spin superradiance.

\section{NANOCLUSTER RADIATION}

The total intensity of spin radiation for the system of magnetic nanoclusters is the dipole radiation intensity

$$
I_{S}(t)=\frac{2 \mu_{0}^{2}}{3 c^{3}}\left|\sum_{i}\langle\ddot{\mathbf{S}}(t)\rangle\right|^{2}
$$

where, as usual, the dots mean time differentiation. This yields

$$
I_{S}(t)=\frac{2 \mu_{0}^{2} \omega_{0}^{4}}{3 c^{3}} N^{2} S^{2}[1-A s(t)]^{4} w(t)
$$

Here $\mu_{0}$ is the average nanocluster magnetic moment and $A$ is the anisotropy parameter defined by the expressions

$$
\begin{gathered}
A \equiv \frac{\omega_{A}}{\omega_{0}}, \quad \omega_{A} \equiv \omega_{D}+\frac{1}{2} \omega_{2}, \\
\omega_{D} \equiv(2 S-1) D, \quad \omega_{2} \equiv(2 S-1) D_{2} .
\end{gathered}
$$

The superradiant pulse is accompanied by the spin reversal. The reversal time is $t_{\text {rev }} \sim 10^{-12} \mathrm{~s}$. The peak of radiation intensity, depending on the number of nanoclusters $N \sim 10^{14}-10^{20}$, reaches $I_{S} \sim 1-10^{12} \mathrm{~W}$. The typical superradiant pulse is shown in Fig. 4, where $I(t)$ is presented in dimensionless units, so that

$$
I(t) \equiv[1-A s(t)]^{4} w(t) .
$$

The possibility of obtaining relatively high radiation intensity is due to the high spin values of the nanoclusters, $S=10^{3}$.

\section{CONCLUSION}

We have shown that coherent radiation can be realized with the ensemble of quantum dots as well as with the assembly of magnetic nanoclusters. In both the cases, it is possible to represent the evolution equations in a similar mathematical form, as the equations of pseudospin or spin variables. Therefore, these equations can be solved by employing the method of scale separation. By direct numerical simulations of the equations of motion it is shown that this approach provides accurate description of the dynamic phenomena.

Despite the formal similarity of the evolution equations, the underlying physics for quantum dots and magnetic nanoclusters is drastically different. For quantum dots, the coherence develops due to the photon exchange through the common radiation field, that is, due to the Dicke effect. While for magnetic nanoclusters the arising coherence is caused by the resonator feedback field, that is, due to the Purcell effect. The coherent spin motion is unachievable for spin systems without a resonator [2834].

The appearance of coherence in quantum dots has been detected experimentally [38], though the realization of pure superradiance, to our knowledge, has not been accomplished.

There have been attempts to observe radiation from magnetic molecules $\mathrm{Mn}_{12}$ [39] and $\mathrm{Fe}_{8}$ [40]. However, in these attempts, no resonator was involved. But, as is emphasized above, without a resonator no coherence in the spin motion can exist. Because of this, such a radiation from neither magnetic molecules nor magnetic nanoclusters has yet been experimentally detected.

\section{ACKNOWLEDGMENTS}

Financial support from the Russian Foundation for Basic Research is acknowledged. 

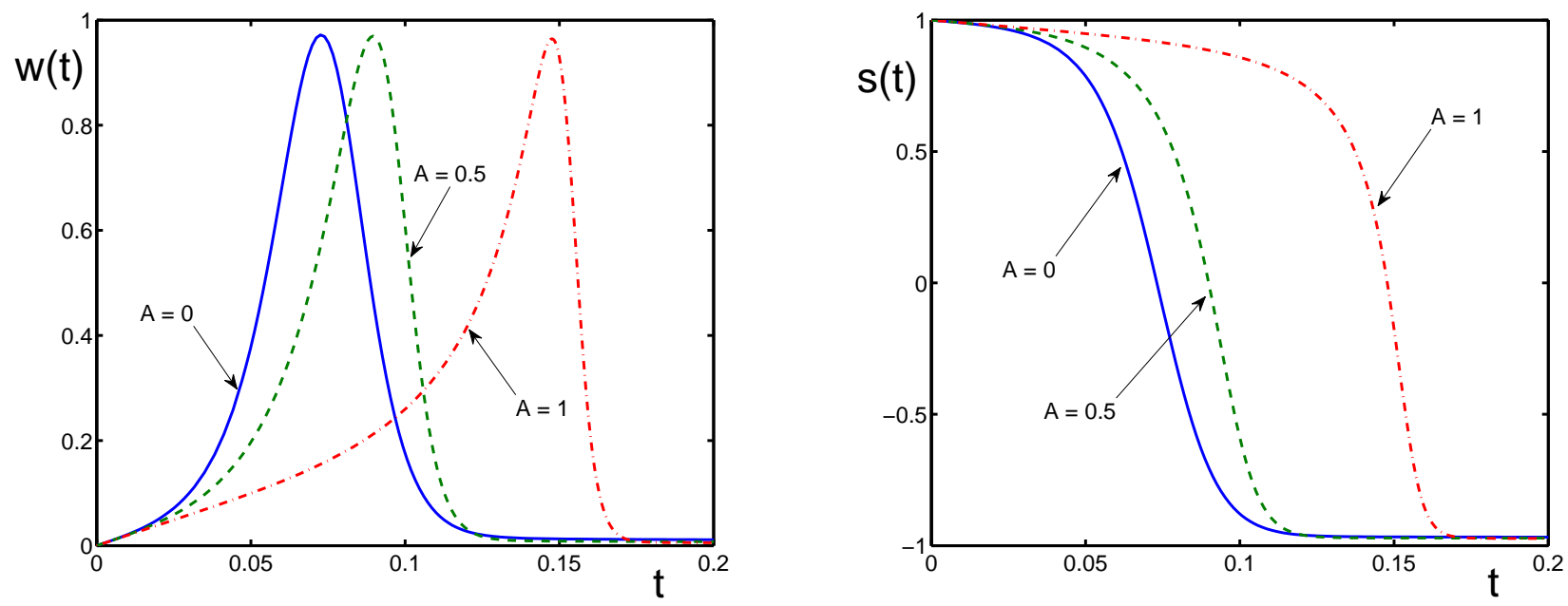

FIGURE 3. Pure spin superradiance, under initial conditions $w_{0}=0, s_{0}=1$. Coherence intensity $w(t)$ and spin polarization $s(t)$ as functions of time (in units of $1 / \gamma_{2}$ ) for $\gamma=10 \gamma_{2}, \gamma_{1}=10^{-3} \gamma_{2}, g=100$, and for different anisotropy parameters: $A=0$ (solid line), $A=0.5$ (dashed line), and $A=1$ (dashed-dotted line).

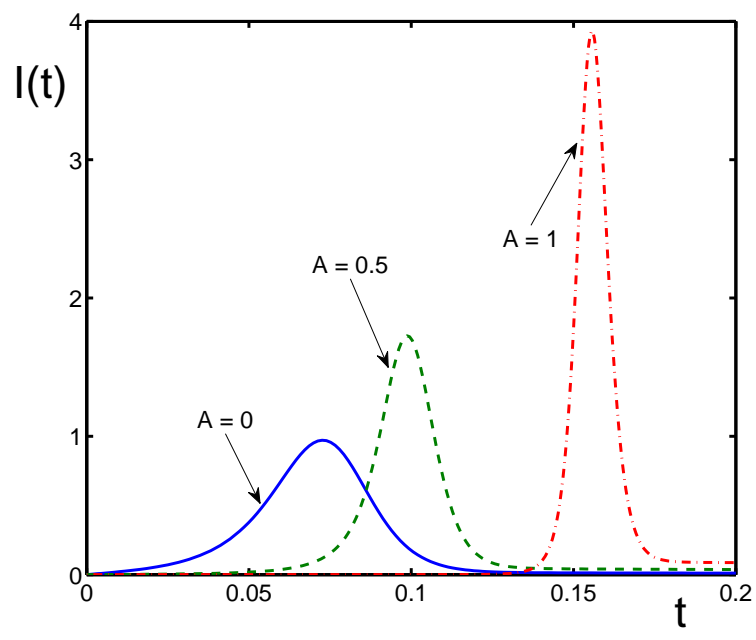

FIGURE 4. Pure spin superradiance, under initial conditions $w_{0}=0, s_{0}=1$. Dimensionless radiation intensity $I(t)$, as functions of time (in units of $1 / \gamma_{2}$ ) for the same parameters as in Fig.3, and for different anisotropy parameters: $A=0$ (solid line), $A=0.5$ (dashed line), and $A=1$ (dashed-dotted line).

\section{REFERENCES}

1. L. Allen, and J. H. Eberly, Optical Resonance and Two-Level Atoms, Wiley, New York, 1975.

2. A. V. Andreev, V. I. Emelyanov, and Y. A Ilinsky, Cooperative Effects in Optics, Institute of Physics, Bristol, 1993.

3. R. H. Dicke, Phys. Rev. 93, 99 (1954).
4. E. M. Purcell, Phys. Rev. 69, 681 (1946).

5. L. P. Kouwenhoven, D. G. Austing, and S. Tarucha, Rep. Prog. Phys. 64, 701 (2001).

6. S. M. Riemann and M. Mannien, Rev. Mod. Phys. 74, 1283 (2002).

7. C. Yannouleas and U. Landman, Rep. Prog. Phys. 70, 2067 (2007).

8. J. L. Birman, R. G. Nazmitdinov, and V. I. Yukalov, Phys. Rep. 526, 1 (2013).

9. M. Singh, V. I. Yukalov, and W. Lau, in Nanostructures: Physics and Technology, edited by Z. Alferov and L. Esaki, Ioffe Institute, St. Petersburg, 1998, p. 327.

10. V. I. Yukalov and E.P. Yukalova, Phys. Rev. B 81, 075308 (2010).

11. V. I. Yukalov and E. P. Yukalova, Phys. Part. Nucl. 31, 561 (2000).

12. V. I. Yukalov, Laser Phys. 3, 870 (1993).

13. V. I. Yukalov, Phys. Rev. Lett. 75, 300 (1995)

14. V. I. Yukalov, Laser Phys. 5, 970 (1995).

15. V. I. Yukalov, Phys. Rev. B 53, 9232 (1996).

16. V. I. Yukalov, Eur. Phys. J. D 13, 83 (2001).

17. V. I Yukalov J. Mod. Opt. 35, 35 (1988).

18. V. I Yukalov J. Mod. Opt. 37, 1361 (1990).

19. V. I. Yukalov, Phys. Lett. A 278, 30 (2000).

20. V.I. Yukalov, Phys. Lett. A 284, 91 (2001).

21. V. I. Yukalov, Physica A 291, 255 (2001).

22. B. Barbara, L. Thomas, F. Lionti, I. Chiorescu, and A. Sulpice, J. Magn. Magn. Mater. 200, 167 (1999).

23. W. Wernsdorfer, Adv. Chem. Phys. 118, 99 (2001).

24. J. Ferre, Topics Appl. Phys. 83, 127 (2002).

25. V. I. Yukalov and E. P. Yukalova, Phys. Part. Nucl. 35, 348 (2004).

26. V. I. Yukalov and E. P. Yukalova, J. Phys. Conf. Ser. 393, 012004 (2012).

27. V. I. Yukalov, Laser Phys. 2, 559 (1992).

28. V. I. Yukalov, Laser Phys. 8, 1089 (2002).

29. V. I. Yukalov, Phys. Rev. B 71, 184432 (2005). 
30. V. I. Yukalov, and E. P. Yukalova, Eur. Phys. Lett. 70, 306 (2005).

31. V. I. Yukalov and E. P. Yukalova, Laser Phys. Lett. 2, 302 (2005).

32. V. I. Yukalov and E. P. Yukalova, Laser Phys. Lett. 2, 356 (2005).

33. V. I. Yukalov and E. P. Yukalova, Laser Phys. Lett. 8, 804 (2011).

34. V. I. Yukalov and E. P. Yukalova, J. Appl. Phys. 111, 023911 (2012).

35. T. S. Belozerova, V. K. Henner, and V. I. Yukalov, Phys. Rev. B 46, 682 (1992).

36. V. I. Yukalov, V. K. Henner, P. V. Kharebov, and E. P. Yukalova, Laser Phys. Lett. 5, 887 (2008).

37. P. V. Kharebov, V. K. Henner, and V. I. Yukalov, J. Appl. Phys. 113, 043902 (2013).

38. M. Scheibner, T. Schmidt, L. Worshech, A. Forchel, G. Bacher, T. Passow, and D. Hommel, Nature Phys. 3, 106 (2007).

39. M. Bal, J. R. Friedman, K. Mertes, W. Chen, E. M. Rumberger, D. N. Hendrickson, N. Avraham, Y. Myasoedov, H. Shtrikman, and E. Zeldov, Phys. Rev. B 70, 140403 (2004).

40. O. Shafir and A. Keren, Phys. Rev. B 79, 180404 (2009). 